\title{
Designing a Mask Programmable Matrix for Sequential Circuits
}

\author{
Fernanda Lima, Marcelo Johann, José Güntzel, Eduardo D’Avila, Luigi \\ Carro, Ricardo Reis \\ Universidade Federal do Rio Grande do Sul - Instituto de Informática \\ Caixa Postal 15064 CEP $91501-970$ - Porto Alegre - RS - Brasil \\ e-mail: <fglima, guntzel, johann, carro, reis>@inf.ufrgs.br \\ phone: + 5551 316-7036 fax: +55 51 319-1576
}

Key words: programmable logic, gate arrays, universal logic gates.

Abstract: This paper proposes the use of Universal Logic Gates (ULGs) as basic elements for masked programmable master-slices customizable by the topmost metal layer. This new approach called Maragata combines the efficiency of MPGAs with the flexibility of FPGA architecture. Due to the intensive use of processor-like blocks in current VLSI circuits, ULGs were developed considering the implementation of sequential circuits. A set of ULGs were studied and designed for CMOS technology. Area comparison was accomplished by mapping various combinational and sequential circuits into ULGs master-slices and to a gate array master-slice called Ágata. Results show that significant area gain and connection reduction can be achieved in this new approach.

\section{INTRODUCTION}

Rapid prototyping is the key to quick turnaround in a product development process. Today's fast paced design cycles require the availability of early silicon and the flexibility of ramping to any volume production. Field Programmable Gate Arrays (FPGAs) are the most popular solution for the time-to-market because they can provide instant manufacturing and low cost prototyping. Since Xilinx Company [XIL98] 
introduced the FPGA in 1985, many FPGAs have been developed by a number of other Companies like Actel [ACT98], Altera [ALT98].

FPGAs continue to fall short masked gate arrays in performance, density and cost for high volume. Masked Programmable Gate Arrays (MPGAs), on the other hand, have longer turnaround times. New technologies and solutions have emerged to overcome the limitations of FPGAs while maintaining the benefits of traditional gate arrays. One solution is masked gate arrays customizable only by the topmost metal layer [DON93] called Quick Customizable Logic (QCL). Another solution for fast prototyping is the Laser Programmable Gate Array (LPGA). ChipExpress Company [CHI98] offers such programmable gate arrays, which are composed of programmable logic blocks. LPGAs, MPGAs and FPGAs differ significantly in unit price, density, performance and prototyping lead times. Figure 1 shows different logic density and design time tradeoffs.

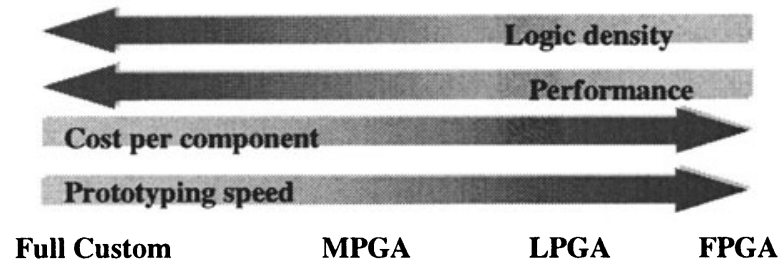

Figure 1. Digital Systems Implementation Options

Present days technology allows the integration of a large number of transistors and the possibility of integrating complete systems on a single chip. In the last 20 years, many technological breakthroughs have led to deep modifications either in our production system, or even in the way people interact with the overall world. Processor-like circuits, on a direct consequence of the integration technology availability, have made a dramatic change in the way systems are designed. The concept of an Application Specific System including microprocessors inside covers a wide range of applications, from portable systems to dedicated embedded control devices or ubiquitous computing.

Aiming at increasing logic density of digital circuits with embedded processors inside, implemented in a programmable matrix, a new methodology based on mask programmable matrix customizable by the top most metal layer is proposed. This new approach is called Maragata [LIM98]. In this methodology the transistor rows are replaced by programmable logic blocks that can be specifically named as Universal Logic Gates (ULGs). Maragata is composed of coarse grain ULGs like in a hard-wired version of a FPGA architecture that combines the efficiency of MPGAs with the flexibility of FPGA architecture. Its ULGs were developed 
considering the implementation of sequential and processor-like circuits, because these ULGs can implement latches or flip-flops with low area cost.

\section{DESIGNING UNIVERSAL LOGIC GATES}

The large flexibility of ULGs justifies its use for building up programmable matrix, particularly when customization is performed by using the topmost metal layer. When a more complex cell is used for building MPGAs, it is possible to optimize silicon area by properly sizing its transistors. Moreover, in such approach the transistor connections as well as small connections are already done. For instance, internal cell transistors that do not have to drive large capacitive loads may be smaller or even of minimum size. Overall timing performance of the cell is assured by sizing output cells as buffers by the time the matrix is designed.

A ULG can be defined as a function $f\left(x_{1}, \ldots, x_{m}\right)$ that can realize some $n-$ input functions $\mathrm{G}\left(\mathrm{y}_{1}, \ldots, \mathrm{y}_{\mathrm{n}}\right)$ connecting $\mathrm{x}_{\mathrm{j}}$ to $1,0, \mathrm{y}_{\mathrm{i}}$ or $y_{i}$, or negating the output of $\mathrm{G}$, where $\mathrm{m}>\mathrm{n}$. While designing a ULG for a specific programmable methodology, different ULG issues must be carefully analyzed. One issue is the functionality. The number of different Boolean logic functions that can be implemented by the cell defines the ULG functionality. The logic block functionality dominates the matrix logic density. Different ULGs are likely to have different amounts of functionality, and varying costs in terms of area and delay. This issue is very important because it will affect the logic density and the amount of routing resources. Two main considerations when selecting a given structure is to see how many $n$-input functions it can implement, and how easy is to implement a latch or flip-flop using all ULG resources. These logic facts must be combined with layout and electrical issues to ensure good performance and cost.

The topology of a ULG directly influences its functionality. Multiplexors, inverters and simple logic gates may be combined together to build ULGs. A set of topologies was studied analyzing their functionality to achieve an optimal ULG. The goal is to find a ULG capable of realizing as many functions as possible without compromising silicon area and performance.

We have done some research to select a good ULG, looking for low granularity, high flexibility and the availability of a technology mapper. Figure 2 shows some ULGs developed to Maragata approach.

The proposed ULGs to Maragata can implement either combinational logic or sequential logic. Most of FPGAs have logic blocks that can implement combinational logic. To implement sequential logic it is 
necessary a flip-flop per logic blocks. When this logic block is used only for combinational logic, the flip-flop area is wasted. The ULG3 can implement a flip-flop master-slave (with set and reset) using its multiplexors. It is necessary two ULG3 to implement one flip-flop and only one CLUS2 to implement the same flip-flop.

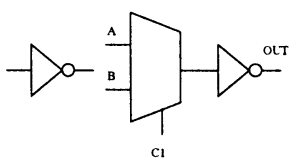

(a) ULG1

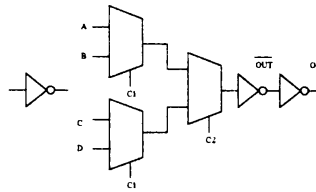

(b) ULG3

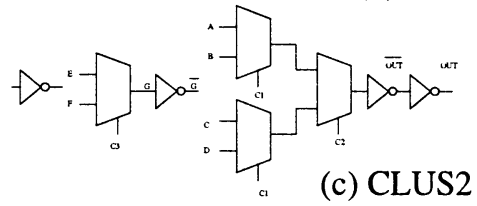

(c) CLUS2

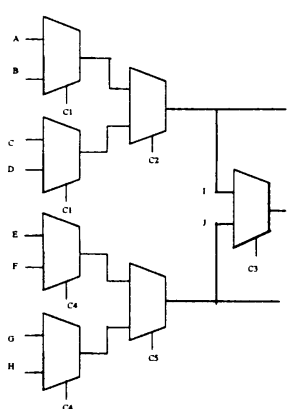

(d) CLUS3

Figure 2. ULGs developed to Maragata

These ULGs developed to Maragata approach were compared to some ULGs developed to commercial FPGAs and LPGAs. Figure 3 shows some ULGs proposed in [LIN97] and in FPGA families like Actel and ChipExpress.

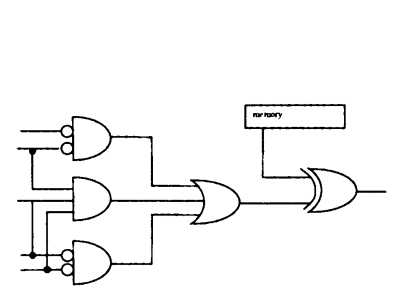

(a)

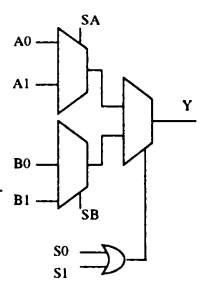

(b)

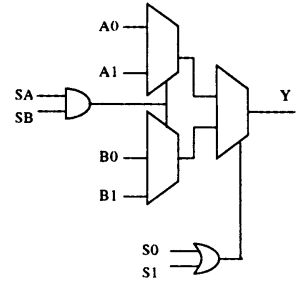

(c)

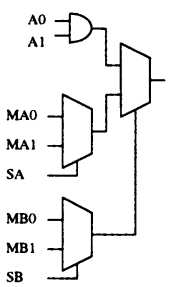

(d)

Figure 3. ULGs proposed in [LIN97] (a), Actel (b)(c) and ChipExpress (d) logic blocks

The ULGs presented in figure 2 and in figure 3 were analyzed using the Programa_de_TV tool [LIM99b] and their functionality was obtained. This program is able to realize all the NPN operations $\left(\mathrm{N}_{\mathrm{I}}, \mathrm{P}\right.$ and $\left.\mathrm{N}_{\mathrm{O}}\right)$ over any $\mathrm{n}$ input Lookup Tables, where $\mathrm{n}$ can be 2,3 or 4 . From the ULG description it is possible to identify all NPN classes that can be realized by this ULG. The graph on figure 4 shows the number of n-input NPN classes implemented by these ULGs. Figure 4 also shows the granularity of ULGs. The relative area indicates this granularity. The number of transistors was calculated based on multiplexors composed of transmission gates. It is necessary to use 6 transistors to implement one 2:1 multiplexor. For example, ULG3 in the $\mathrm{x}$ axis implements all 2-input and 3-input logic functions, few 4-input logic 
functions and occupies a small fraction of the largest studied ULG area (CLUS3). This one, although with larger area, implements all 4-input logic functions. ULG3 is the best tradeoff among number of logic functions, area and possibility of implementing a full flip-flop.

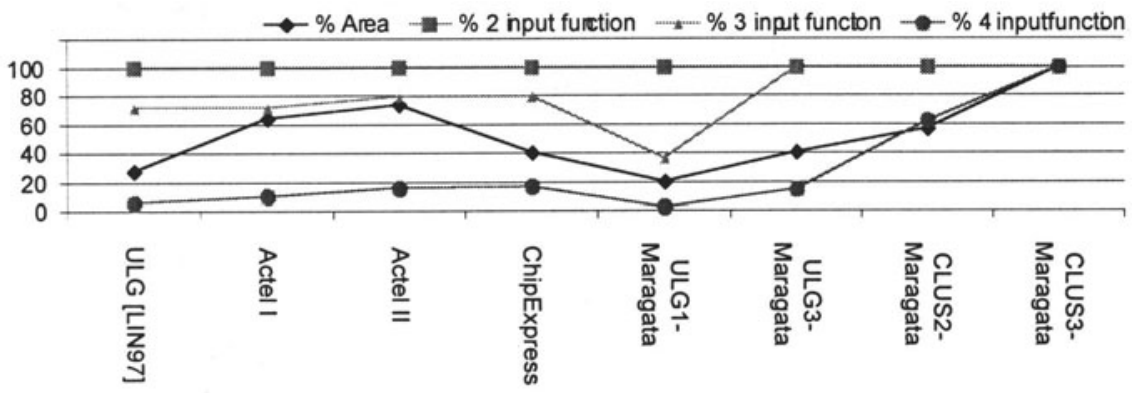

Figure 4. Percentage of number of NPN classes implemented by some ULGs presented in fig. 2 and fig 3, and the percentage of area, respectively

The multiplexers of Maragata ULGs were implemented by using transmission-gates rather than by CMOS static gates, to minimize not only transistor count, but power dissipation as well. In order to achieve minimum layout area, minimum width transistors were used whenever it is possible. In each ULG output transistors were sized to work as buffers. Internal fixed and customizable cell connections may contribute to reduce channel routing complexity.

Table 1 shows the number of transistors and area for all developed ULGs. They have been developed in $0.8 \mu \mathrm{m}$ double metal layer CMOS technology. All the customizable connections are done over the ULG without using the routing channel. The first metal layer was used for internal connections, while the second one was reserved for customization. Table 1 also presents the area comparison for a master-slave flip-flop implemented into different ULGs. The cell CLUS3 can either implement 1 bit register or a D flip-flop.

Table 1. ULGs Characteristics

\begin{tabular}{lcccc}
\hline ULG & \# transistors & Area $\left(\mu \mathrm{m}^{2}\right)$ & $\begin{array}{c}\text { \# ULGs to implement a } \\
\text { flip-flop }\end{array}$ & $\begin{array}{c}\text { Area }\left(\mu \mathrm{m}^{2}\right) \\
\text { of a flip-flop }\end{array}$ \\
\hline ULG1 & 10 & 1057 & 4 & 4228 \\
ULG3 & 22 & 1922 & 2 & 3844 \\
CLUS2 & 30 & 3000 & 1 & 3000 \\
CLUS3 & 50 & 5000 & 1 & 5000 \\
\hline
\end{tabular}

Figure 5 presents a circuit layout implemented in the Maragata matrix. The customization is done in metal 2. This matrix is composed of 26 rows, 80 pads and has 1040 ULG3s. The matrix area is about $11.03 \mathrm{~mm}^{2}$. Its logic 
density is $2263 \mathrm{tr} / \mathrm{mm}^{2}$. It is important to notice that the routing channel takes a significant area. By reducing connections one can expect a large reduction in the total matrix area.
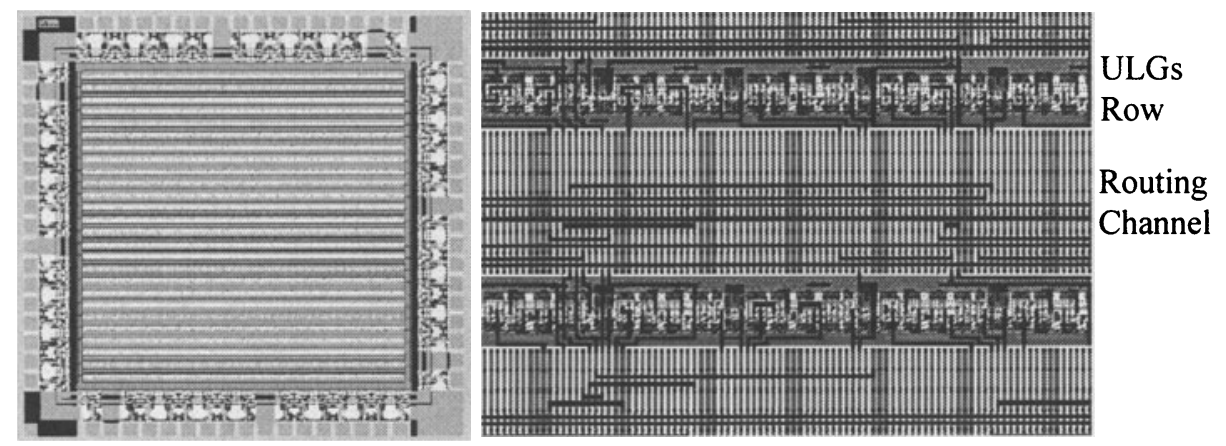

Figure 5. Matrix layout (the routing channel, the ULG rows and the customization in metal 2)

\section{AREA AND CONNECTION COMPARISON}

In order to evaluate the silicon area gain at Maragata approach, combinational and sequential circuits were mapped into conventional MPGA library elements and to ULGs from Maragata. SIS [SEN92] was used for logic simplification and technology mapping. In case of ULGs, the n-LUT mapper (SIS) was used (xl_part_coll -m -g 2 -n n; xl_coll_ck $-\mathrm{n} n$; $\mathrm{xl}$ partition -m -n n; simplify; $\mathrm{xl}$ _imp - $\mathrm{n} \mathrm{n}$; $\mathrm{xl}$ _partition - $\mathrm{t}-\mathrm{n} \mathrm{n}$; $\mathrm{xl}$ _cover -e $30-\mathrm{u} 200-\mathrm{n} n$; xl_coll_ck -n n).

The conventional MPGA approach chosen for benchmarking is a single metal layer customizable, based on transistor rows, refered to as Ágata [CAR96]. It has been designed using the same $0.8 \mu \mathrm{m}$ double metal layer CMOS technology. Its library of personalization patterns has 13 logic functions: inverter, 2-, 3- and 4-input nands and nors, 2-input xor and nxor, 2 to 1 and 4 to 1 multiplexers. There are also two latches and one D masterslave flip-flop with set and reset. In the case of Ágata, it was used the command map -m 0.

Table 2 shows some area and connections results for some combinational and sequential circuits implemented into ULG3. Some of these circuits are from the MCNC'91 benchmark. Previous research [LIM99a] showed that the most appropriated ULG in terms of area and number of connection efficiency is the ULG3.

The area result presents only the amount of active area (cell area without taking account the routing channel) used in each design. Connectivity plays an important role in QCL like designs, since the number of possible connections is fixed. For the Ágata approach there is a complete router 
working [JOH97], and this same router is used in the Maragata approach. Since the ULG can embed more logic in its core, much less internal connections are needed in the Maragata case when compared to Ágata, as it is showed in table 2 .

Table 2. Area comparison between Ágata and Maragata ULGs for combinational and sequential circuits mapped using SIS

\begin{tabular}{ccccccr}
\hline Circuits & \multicolumn{2}{c}{ MAP Ágata } & \multicolumn{2}{c}{ ULG3 (3-LUT) } & \multicolumn{2}{c}{ Comparison } \\
\hline & CX & Area & Area & CX & \%A & $\%$ CX \\
Booth & 433 & 350232.4 & 282534 & 257 & -19 & -41 \\
Booth12 & 690 & 545349.2 & 417074 & 380 & -24 & -45 \\
Booth16 & 936 & 731685.2 & 574678 & 526 & -21 & -44 \\
Boothp8 & 829 & 537555.6 & 461280 & 475 & -14 & -43 \\
Bisect8 & 919 & 610075.2 & 551614 & 549 & -10 & -40 \\
Desloca & 379 & 317387.2 & 251782 & 224 & -21 & -41 \\
Descola12 & 514 & 764208.8 & 524706 & 535 & -31 & 4 \\
8051_control & 125 & 72194.4 & 78802 & 92 & 9 & -27 \\
8051_valida & 267 & 150242.4 & 188356 & 200 & 25 & -25 \\
8051_stateg & 143 & 108940.0 & 105710 & 99 & -3 & -31 \\
8051_multipl. & 885 & 594790.8 & 517018 & 511 & -13 & -42 \\
Raiz_bs & 664 & 678666.8 & 526628 & 383 & -22 & -42 \\
Raiz_ds & 796 & 790859.4 & 601586 & 453 & -24 & -43 \\
Raiz4q & 5102 & 4733799.6 & 3865142 & 3090 & -18 & -39 \\
\hline
\end{tabular}

Results shows that area reduction may be achieved when the Maragata approach is used to implement a microprocessor (8051) and arithmetic functions like multipliers (booth, boothp, desloca, and bisect) and square extractor (raiz_bs, raiz_ds and raiz4q) using the ULG3 cell. The use of ULGs resulted in area gains around $20 \%$ for almost all examples. Only two circuits implemented in Ágata presented a smaller area instead of implementing in Maragata. For almost all circuits implemented using ULG3, the number of internal connections has been reduced around $40 \%$. None of these circuits presented a simultaneous increase in area and connection.

\section{CONCLUSIONS}

The applicability of ULGs to Quick Customizable Logic (QCL) masterslices has been presented. Comparisons in terms of area gain were developed by mapping some small and medium combinational and sequential circuits. For these circuits, the use of ULGs resulted in area gains around $20 \%$ for combinational and sequential circuits. The number of required connections for different examples was also calculated. Results shows that the Maragata 
approach leads to effective reduction in the number of connections. These area and connection gains can represent a logic density improvement because more connections can be done in the same routing channel.

Current works include the investigation of more appropriate mapping algorithms for ULGs master-slices, to further improve results in both combinational and sequential circuit cases.

\section{REFERENCES}

[ACT98] ACTEL CORPORATION. Introduction to Actel FPGA Architecture. http://www.actel.com. (Nov.1998).

[ALT98] ALTERA CORPORATION. Data Sheet. http://www.altera.com (Nov. 1998).

[BET98] BETZ, Vaughn; ROSE, Jonathan. How Much Logic Should Go in an FPGA Logic Block? IEEE Design \& Test of Computer. New York, v. 15, n. 1, 1998. http://www.eecg.utoronto.ca/ jayar/ (Aug. 1998).

[CAR96] CARRO, L.; et al. An environment to Design Digital Circuits Based on the Brazilian Gate-Array. In: WORKSHOP IBERCHIP, 2., 1996. Memorias..., São Paulo, [s.n.], p.198, Feb. 1996.

[CHI98] CHIP EXPRESS CORPORATION. Data Sheet. http://www.chipexpress.com (Nov. 1998).

[DON93] DONG, S. K., et al. Two channel Routing Algorithms for Quick Customized Logic. In: EDAC, 1993. Proceedings... Los Alamitos : IEEE Computer Society, 1993. 2 V. p. 122-126, 1993.

[HOP99] HOPKIN, Vince. Programmable Device or Gate Array? http://www.isdmag.com (Jan. 1999).

[JOH97] JOHANN, M.; CARRO, L.; REIS, R. Functional Design of GAROTA: Gate Array Router of ÁGATA System. In: Brazilian Symposium on Integrated Circuit Design, 10., 1997, Gramado, RS. p. 21-30. Proceedings... Porto Alegre: CPGCC da UFRGS. 1997.

[LIM98] LIMA, Fernanda G.; et al. Improving Logic Density of QCL Masterslices by Using Universal Logic Gates. In: Brazilian Symposium on Integrated Circuit Design, 11., 1998, Búzios, RJ. Proceedings... Los Alamitos : IEEE Computer Society, 1998. 250 p.

[LIM99a] LIMA, Fernanda G.; et al. Designing Masked Programmable ULGs for MPGAs. In: WORSHOP IBERCHIP, 4., 1999. Memorias... [LIMA : HOZLO S.R.L.], 1999. http://www.inf.ufrgs.br/ $\sim$ fglima/maragata.html

[LIM99b] LIMA, Fernanda G.; et al. Programa_de_TV Tool. In: SBCCI, 12., Proceedings..., 1999. To be published by IEEE Computer Society.

[LIN97] LIN, Chih-Chang; MAREK-SADOWSKA, Malgorzata; GATLIN, Duane. On Designing Universal Logic Gate and their Application to FPGA Design. IEEE Transactions on Computer-Aided Design of Integrated Circuits and Systems. New York, v. 16, n. 5, May 1997.

[SEN92] SENTOVICH, E.; et al. SIS: A System for Sequential Circuit Synthesis. Memorandum No. UCB/ERL M92/41. May. 1992.

[XIL98] XILINX CORPORATION. Devices Data Sheet. http://www.xilinx.com. (Nov. 1998). 